\title{
Multimodal biometrics with serial, parallel and hierarchical mode at decision level fusion
}

\author{
Priti S. Sanjekar, J. B. Patil \\ Department of Computer Engineering, R. C. Patel Institute of Technology, \\ Shirpur and KBC North Maharashtra University, India
}

\begin{tabular}{|c|c|}
\hline Article Info & ABSTRACT \\
\hline Article history: & \multirow{11}{*}{$\begin{array}{l}\text { Biometric based personal authentication is playing a vital role in various } \\
\text { security based applications. This paper presents the effective fusion of } \\
\text { fingerprint, palmprint and iris traits at decision level. Combining different } \\
\text { traits at the decision level is a challenging task due to less information } \\
\text { available at this level. The focus of the work is to examine the performance } \\
\text { of multimodal biometrics at decision level fusion in three different i.e. serial, } \\
\text { parallel and hierarchical modes of operation. Serial mode is performed by } \\
\text { taking unimodals serially while parallel mode of operation is carried out by } \\
\text { processing all modals simulatenously using Majority Voting Rule and the } \\
\text { hierarchical mode of operation is performed with proper combination of } \\
\text { traits in parallel and serial mode using AND and OR rule. The experiments } \\
\text { are performed on } 100 \text { different users from publically available FVC2006 } \\
\text { fingerprint database, CASIA V1 palmprint database and IITD iris database. } \\
\text { The experimental results suggest that proper fusion of different traits in } \\
\text { hierarchical way can give best performance even at decision level fusion as } \\
\text { compared to serial and parallel mode of operation. }\end{array}$} \\
\hline Received Dec 26, 2018 & \\
\hline Revised Feb 4, 2019 & \\
\hline Accepted Mar 11, 2019 & \\
\hline Keywords: & \\
\hline Decision level fusion & \\
\hline Fingerprint & \\
\hline Hierarchical mode & \\
\hline Iris & \\
\hline Multimodal biometrics & \\
\hline Palmprint & \\
\hline
\end{tabular}

Copyright $@ 2019$ Institute of Advanced Engineering and Science. All rights reserved.

\section{Corresponding Author:}

Priti S. Sanjekar, Department of Computer Engineering,

R. C. Patel Institute of Technology,

Shirpur and KBC North Maharashtra University, Jalgaon, India.

Email: priti_san2003@yahoo.com

\section{INTRODUCTION}

Automatic personal authentication is becoming an essential part in large variety of security applications. Traditional authentication methods are based on use of key, ID cards, PIN or password. However, with this method there are lots of chances of breaking the security. Biometrics is a pattern recognition system which authenticates the person based on physiological traits like palmprint, iris, fingerprint, retina, face, finger-vein, fingerknuckle or behavioral traits like signature, voice, gait etc [1, 2]. These biometric features cannot be misplaced or stolen hence biometrics is frontier to traditional methods of personal authentication. The biometric system which uses single trait for authentication is a unimodal biometrics. However unimodal biometrics faces some problems like spoofing, interclass similarity, intraclass variations, universality. Also no single biometric characteristic can satisfy all the performance requirements of the system [3]. To alleviate the problems of unimodal biometrics; biometric researchers are now moving towards multimodal biometrics.

Multimodal biometrics makes the use of multiple traits for authentication purpose [4]. Multimodal biometrics integrates the information from different traits at various levels. Proper integration of the information which is gained from different modals is very important to get best system performance [5]. Information from different modals can be merged at Feature, Matching score or Decision level. In features level fusion, features of different traits are merged and form a new feature set which is used for 
authentication. In matching score level fusion, matching scores of different modals are combined after normalization of the scores. In decision level fusion, each modal gives its decision and finally by applying rules final decision is made. Feature level fusion is pre-classification information fusion while score level and decision level fusion are post-classification information fusion [6]. From the operational mode point of view, the multimodal biometrics can operate in serial mode, parallel mode or hierarchical mode [7]. In serial mode, based on the output of the one modality decision is taken into account about the consideration of next modality. In parallel mode, information from all modalities is considered simultaneously for processing while in hierarchical mode, the system takes some traits in parallel and some in serial.

Till date many multimodal biometric systems have been developed by many researchers but still right choice of biometric traits, feature extraction algorithm, matcher/classifier, fusion level and mode of operation are some open challenges in multimodal biometrics. In decision level fusion, rules like AND, OR, majority voting, weighted majority voting, k-means, fuzzy logic are used to get final decision of the system [8]. Suneet Narula Garg et al. have given an approach of multimodal biometrics based on fingerprint and iris with decision level fusion. The classification is done with K-NN and Neural classifiers and finally decision is made with AND and OR rule. They reported that the neural classifier with OR rule performs better with $91.5 \%$ accuracy on database of 50 subjects [9].

The Majority Voting rule based decision level fusion is carried out by Vijayalakshmi G. V. et al. for fingerprint, face and iris based system. Majority voting method has given $99.6 \%$ accuracy with database of 20 subjects [10]. Padma Polash Paul et al. have developed the multimodal biometrics based on face, ear and signature with decision level fusion using social network analysis. The accuracy of the system is $100 \%$ at $5 \%$ FAR [11]. H. Benaliouche et al. have given an approach of decision level fusion in fingerprint and iris based multimodal biometrics. They used fuzzy logic and they got $99.9 \%$ accuracy on the database of 50 subjects [12]. Ibrahim A. Saleh et al. have given an approach of iris and signature based multimodal identification biometrics at decision level fusion. Decision fusion is carried out with AND, OR, Weighted Majority Voting and Bayesian decision. Results suggest that OR rule is best for GAR while AND is best for FAR. With the database of 40 subjects they got accuracy of $96 \%$ at $4 \%$ FAR, $99 \%$ at $1 \%$ FAR, $98 \%$ at $2 \%$ FAR and $96 \%$ with $4 \%$ FAR using AND, OR, Weighted Majority Voting, Bayesian decision rule respectively [13]. Mohamad Abdolahi et al. have presented fuzzy logic based decision level fusion for fingerprint and iris based multimodal biometric system. With this fusion at $2 \%$ FAR, $98.3 \%$ accuracy is achieved [14].

Amioy Kumar et al. have applied decision level fusion on palmprint and hand vein based multimodal biometric system with focus on Ant Colony Optimization. They used AND rule for fusion. They have got $99 \%$ recognition with database of 150 subjects and they concluded that Ant Colony Optimization is better than Particle Swarm Optimization [15]. M. Hanmandlu et al. have used hybrid Particle Swarm Optimization for selection of the decision rule. The developed multimodal biometric system is based on palmprint and hand Geometry [16]. Vassilios Chatzis et al. have developed multimodal biometric system based on speech and still images with decision level fusion. They used fuzzy clustering algorithm and experiment was carried on database of 37 subjects, they got $0.6 \%$ FRR at $0.3 \%$ FAR [8]. Zahid Akhtar et al. have worked with parallel mode of operation with matching score level fusion and serial mode in decision level fusion with face and fingerprint traits. They reported that serial mode of operation takes less operational time than parallel mode [17].

Compared to sensor level, feature level and match score level, the fusion at decision level is less addressed in the literature [18]. From literature survey it is observed that most of the previous decision level based multimodal systems work in parallel mode of operation. It is also observed that when multimodal biometrics is based on more than two traits then majority voting rule which is parallel mode of operation has been used by researchers. To the best of our knowledge, hierarchical mode of operation at decision level fusion in fingerprint, palmprint and iris based multimodal biometrics is not yet addressed.

In present work, fingerprint, palmprint and iris are used as biometric traits to develop the multimodal biometric verification system. The information from three different modals is combined at decision level fusion. The aim of this work is to evaluate serial mode by considering modals serially, parallel mode by majority voting rule and hierarchical mode by applying AND rule on two parallel modals first and then depending on this decision from first two modals third modality is considered serially for operation. The focus of the work is on the evaluation of performance of serial, parallel and hierarchical mode of operation at decision level fusion. The rest of the paper is organized as follows: the proposed unimodal and multimodal methodology is described in Section 2, experimental results and discussions are given in Section 3 and finally conclusion is reported in last section. 


\section{PROPOSED METHOD}

The proposed multimodal biometric verification system is based on three traits i.e. fingerprint, palmprint and iris. These traits are chosen for system development because fingerprint is most widely used and accepted trait and palmprint has large area with distinctive features. Third modal i.e. iris is unique to each person; even twins have different irises [7]. This section describes the structure of proposed unimodal and multimodal system.

\subsection{Unimodal Biometrics}

\subsubsection{Fingerprint Verification}

The image based approach is used for fingerprint features extraction. First core point of the fingerprint is detected and area around the core point is considered for feature extraction [19]. The fingerprint features are extracted by applying wavelet transformation which is multi-resolution analysis. The wavelet transform is found to be well-established tool for applications of image processing [20]. The various wavelet components are the scaled and the shifted versions of a mother wavelet [21, 22]. The fingerprint image is decomposed up to 4 levels using Symlet5 wavelet then GLCM of LH, HL and HH parts is computed with 00, 900, 450 and 1350 angle (for HH part GLCM is computed with 450 \& 1350) respectively. From these GLCMs energy and contrast features (32 features) are extracted also wavelet energy of detail components up to fifth level decomposed image is extracted (45 features). Standard deviation and mean of detail component of first level decomposed image are also extracted (6 features). Along with this, gray level co-occurrence matrix of original image is calculated with 00, 900, 450 and 1350 angle and features i.e. energy, contrast, homogeneity, correlation (16 features) are extracted using (1) to (4) respectively.

$$
\begin{aligned}
& \text { Energy }=\sum_{\mathrm{i}, j=1}^{k} \mathrm{P}\left(\mathrm{i}_{i},\right)^{2} \\
& \text { Contrast }=\sum_{\mathrm{i}, \mathrm{j}=1}^{\mathrm{k}}(\mathrm{i}-\mathrm{j})^{2} \mathrm{P}\left(\mathrm{i}_{j} \mathrm{j}\right) \\
& \text { Homogeneity }=\sum_{\mathrm{i}, j=1}^{k} \frac{P\left(i_{i}, j\right)}{1+|i-j|} \\
& \text { Correlation }=\sum_{\mathrm{i}, j=1}^{k} \frac{(\mathrm{i}-\mu \mathrm{i})(\mathrm{j}-\mu \mathrm{j}) \mathrm{P}\left(\mathrm{i}_{i}, \mathrm{j}\right)}{\sigma_{\mathrm{i}} \sigma_{\mathrm{j}}}
\end{aligned}
$$

In this way total 99 features are extracted from each fingerprint. In verification stage, matching of the fingerprint is performed using Euclidean distance using (5). The performance of the fingerprint verification is measured in terms of Equal Error Rate (\%ERR). In fingerprint unimodal verification, equal error rate of $5.6 \%$ is achieved.

$$
\mathrm{ED}=\sqrt{\left(\sum_{\mathrm{i}=1}^{\text {no. of features }}\left(f e a_{k_{i} \mathrm{i}}-f e a_{\mathbb{l}_{i} \mathrm{i}}\right)^{2}\right)}
$$

\subsubsection{Palmprint Verification}

In order to extract the features from palmprint, the wavelet transformation is carried out on the preprocessed palmprint image. First Region of Interest of palmprint is extracted [23]. Then it is wavelet decomposed at first level using BiorSplines 2.4 wavelet. Local statistical analysis is performed on wavelet decomposed palmprint image. Total 400 local statistical features are extracted from palmprint by block processing. The mean of each sub region of the palm is extracted as local statistical features of palmprint using (6).

$$
\operatorname{mean}(\mathrm{m})=1 / \mathrm{N}^{2} \sum_{\mathrm{i} j=1}^{\mathbb{N}} \mathrm{I}\left(\mathrm{i}_{\mathrm{s}} \mathrm{j}\right)
$$

In verification stage, Euclidean distance is used for matching purpose as with (5). Then the performance of the palmprint unimodal system is measured in terms of equal error rate. The equal error rate of $5.5 \%$ is achieved with palmprint unimodal verification system. 


\subsubsection{Iris Verification}

The process of the iris feature extraction is also based on wavelet transformation. Features are extracted from preprocessed i.e. normalized iris image, IITD database consist of normalized iris images [24]. The wavelet transformation up to four levels is carried out using Haar wavelet on normalized iris images. After fourth level of decomposition the wavelet coefficients of LL, LH, HL and HH components are considered as features of iris. The coefficients of the LL, LH, HL and HH sub bands are converted into 324 bits binary code using (7), where $\mathrm{V}$ is feature vector and $\mathrm{V}$ (i) is ith bit of feature vector.

$$
\begin{aligned}
& \mathrm{V}(\mathrm{i})=1, \quad \text { if } \mathrm{V}(\mathrm{i})>0 \\
& \mathrm{~V}(\mathrm{i})=0, \quad \text { otherwise }
\end{aligned}
$$

In matching stage, the iris matching is performed using Hamming distance with (8). The performance of the system is measured in terms of equal error rate. The equal error rate of $1.5 \%$ is achieved with haar wavelet in iris verification system.

$$
\mathrm{HD}(\mathrm{p}, \mathrm{q})=1 / \mathrm{N} \sum_{j=1}^{N}\left(p_{j} \oplus q_{j}\right)
$$

In this way all three unimodal systems are developed and these are combined at decision level fusion to develop multimodal biometric verification system.

\subsection{Multimodal Biometrics with Decision Level Fusion}

The decision level fusion is also called as abstract level fusion. As stated in Section 1, in decision level fusion the individual modality give its decision and based on their individual decision, final decision is given by the multimodal system with the help of fusion rules. In verification, system gives final decision as accept /reject and in identification system it gives identity of the person. In present work, the performance of the fingerprint, palmprint and iris based multimodal biometrics is measured with serial, parallel as well as hierarchical mode of operation at decision level fusion.

\subsubsection{Serial Mode of Operation}

In serial mode of operation, modals are considered one after another. The final decision is accepted depends upon acceptance of any one of the modal otherwise it is rejected. First fingerprint modal is taken as an input. If fingerprint accepts the person then person is treated as genuine otherwise next modal i.e. palmprint is serially taken as an input. If palmprint accepts the person then person is treated as genuine otherwise final modal i.e. iris is taken as an input in serial manner. If iris accepts the person then person is treated as genuine otherwise treated as imposter. Figure 1 shows block diagram of multimodal biometric system in serial mode of operation at decision level fusion. If any one of the three modals accepts the person then final decision is accept otherwise person is rejected by considering imposter.

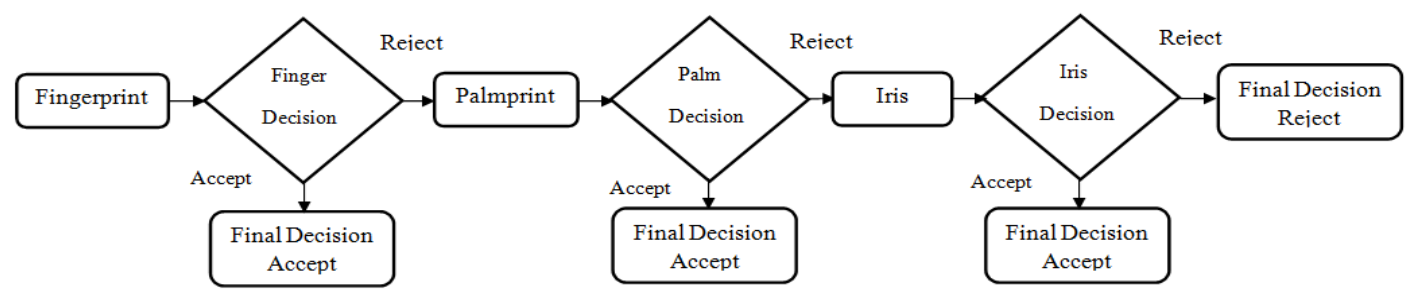

Figure 1. Multimodal biometric verification in serial mode of operation at decision level fusion

\subsubsection{Parallel Mode of Operation}

In parallel mode of operation, decisions from individual unimodal verification systems i.e. fingerprint, palmprint and iris are fused using majority voting rule. Majority voting rule is frequently used in literature for more than two modals so in present work it is used for parallel mode. In majority voting rule, the final decision is based on majority of decision given by different traits and it works in parallel mode. In parallel mode, all the traits are processed simultaneously to get final decision of the system. Figure 2 shows block diagram of our multimodal biometric system in parallel mode of operation at decision level fusion. 


\subsubsection{Hierarchical Mode of Operation}

In hierarchical mode, the some traits work in parallel mode and some in serial modes of operation. First fingerprint and palmprint verification systems work in parallel mode of operation. The individual decision given by fingerprint and palmprint system are combined using AND fusion rule. In AND rule, person is accepted if both systems accept the person. If in the parallel mode of fingerprint and palmprint the person is rejected then third modal i.e. iris is processed in serial manner. Finally decision given by the iris is considered as a final decision. The block diagram of proposed multimodal biometric system in hierarchical mode of operation at decision level fusion is shown in Figure 3.

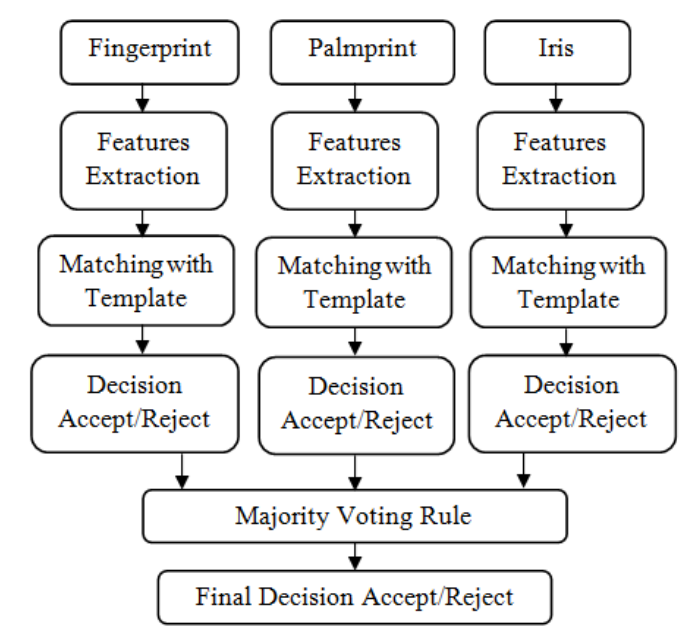

Figure 2. Multimodal biometric verification in parallel mode of operation at decision level fusion

In this way parallel, serial and hierarchical mode of operation are performed at decision level fusion and performance of the system is measured. Next section covers the databases and experimental results of the system.

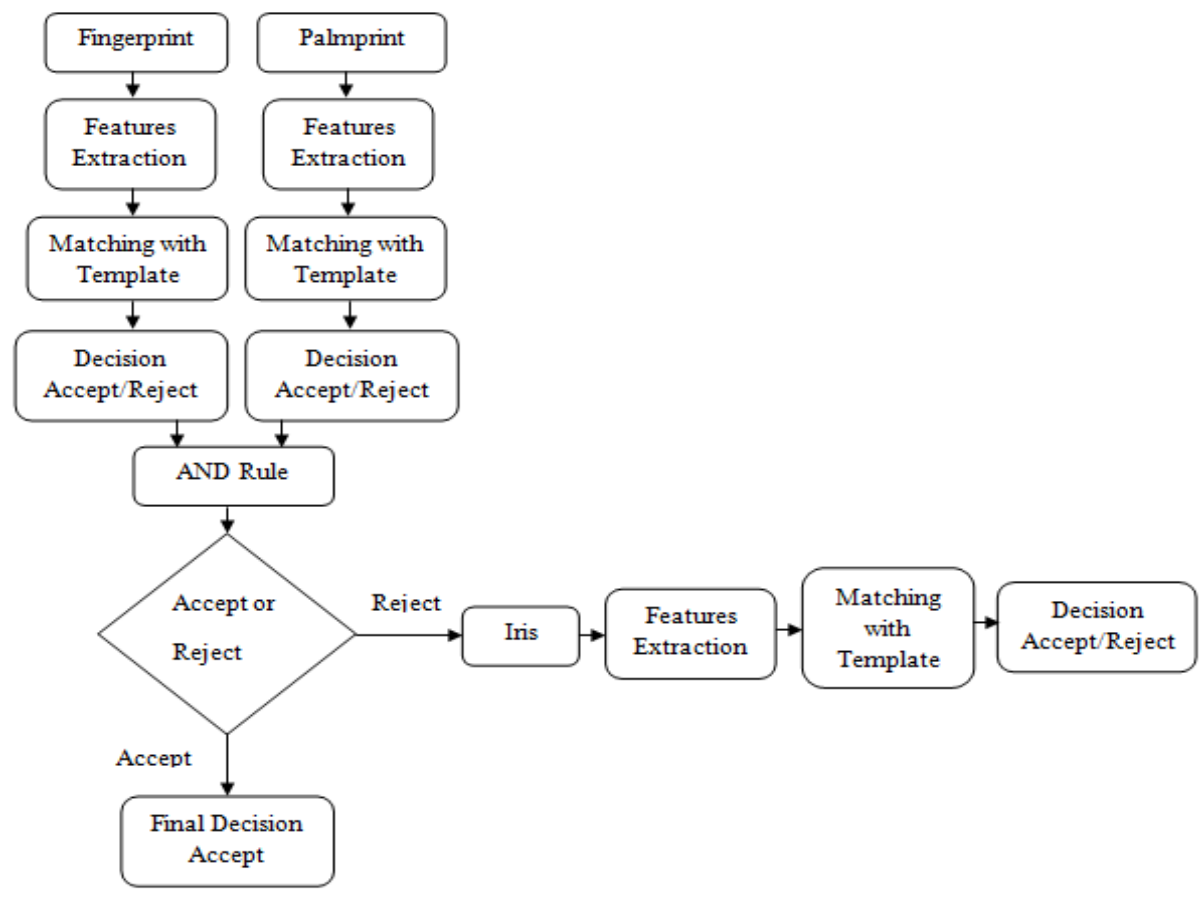

Figure 3. Multimodal biometric verification in hierarchical mode of operation at decision level fusion 


\section{RESULTS AND DISCUSSION}

The proposed work is implemented in MATLAB. In present work, multimodal database used in the experiment is heterogeneous database which is formed from three different unimodal databases i.e. fingerprint, palmprint and iris. The user in the one database is paired with user from other database. The FVC 2006 database is used for fingerprint. This database is collected by Biometric System Lab (BIOLAB), University of Bologna [25]. The fingerprint experimentations are performed on fingerprint images of 100 different subjects form DB2_A set. For palmprint, publically available CASIA Palmprint V1 Database collected by the Chinese Academy of Sciences, Institute of Automation (CASIA) is used [26]. The palmprint experimentations are performed on images of 100 different subjects. Finally for iris, IITD Iris Image Database Version 1.0 acquired in the Biometrics Research Laboratory is used. The normalized iris images are also provided in the database [24]. The normalized iris images of 100 different subjects are used in the experimentations of iris. Here, for each trait 5 images of 100 different subjects are considered for experimentation. Three images of each subject are considered for training while remaining images are used for testing purpose.

The performance of unimodal as well as multimodal system is measured in terms of False Acceptance Rate (FAR), False Rejection Rate (FRR), Genuine Acceptance Rate (GAR) and Equal Error Rate (EER). With lower EER system gives better performance [27]. In unimodal experimentations, EER of 5.6\% is achieved using sym5 wavelet for fingerprint while for palmprint we achieved 5.5\% EER with bior2.4 wavelet and finally for iris EER of $1.5 \%$ is achieved with the use of haar wavelet. Figure 4, Figure 5 and Figure 6 show the Equal Error Rate obtained for unimodal systems. From equal error rates of unimodal traits it is observed that the iris has given more accuracy than fingerprint and palmprint. Receiver Operating Characteristic i.e. ROC defines plot of FAR vs GAR, ROC of the unimodal fingerprint, palmprint and iris systems at different values of FAR are shown in Figure 7.

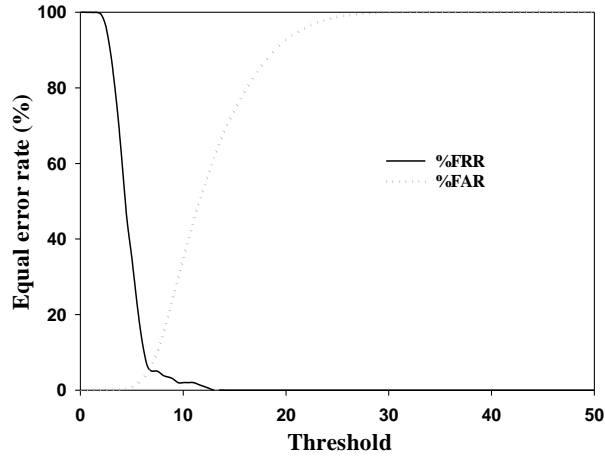

Figure 4. Performance of fingerprint verification

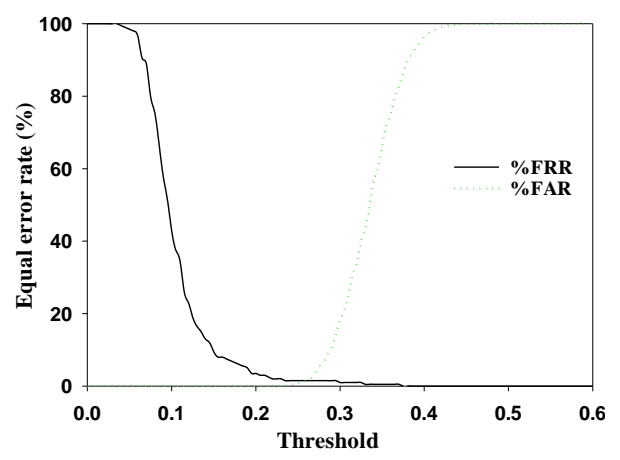

Figure 6. Performance of iris verification

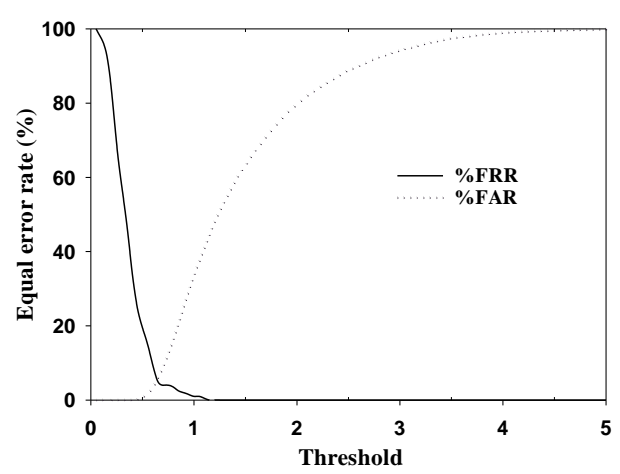

Figure 5. Performance of palmprint verification

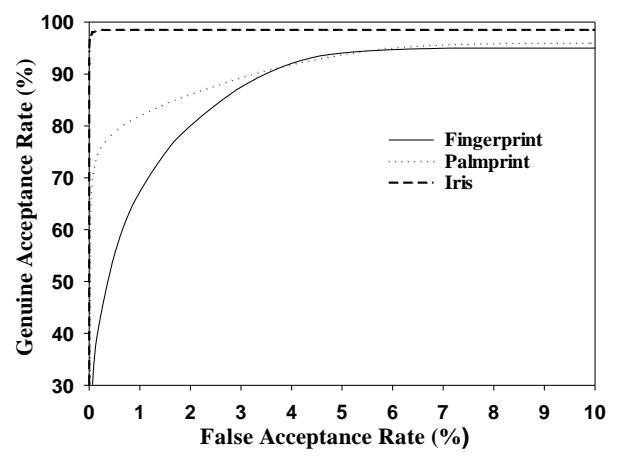

Figure 7. Performance of the unimodal systems

Further, the decision level fusion is carried out in the development of multimodal system and the performance of the system is measured for serial, parallel as well as hierarchical mode of operation. In serial mode of operation $93 \%$ GAR is achieved at $0.01 \%$ FAR. In parallel mode, all the three traits are processed 
simultaneously and their majority decision is considered as a final decision. With parallel mode of operation 92.5\%GAR is achieved at $0.01 \%$ FAR. Generally, use of only AND rule gives better i.e. low False Acceptance Rate (FAR) but False Rejection Rate (FRR) is very high may be higher than individual biometric trait while use of only OR rule improves Genuine Acceptance Rate (GAR) but it also increases the False Acceptance Rate (FAR) [28]. So using the simply AND or OR rule in decision level fusion may not be practically feasible because the system should give high GAR at very low FAR. With this observation, in present work the optimal combination of AND and OR rule is performed at hierarchical mode of operation to achieve high GAR at very low FAR. In present work, out of three traits fingerprint and palmprint have low accuracy as compare to iris trait. The combination of two weak matchers results in good performance [7]. So in the first stage of hierarchical mode, fingerprint and palmprint are combined in parallel mode using AND rule which tries to minimize False Acceptance Rate i.e. FAR. If first stage of hierarchical mode accepts the person then there no need for iris processing, but if first stage results is the rejection then second chance is given to person with the third trait i.e. iris which is processed serially with OR rule and it results final decision of the system. With hierarchical mode, system gives $99 \%$ GAR at $0.01 \%$ FAR and obtained the Equal Error Rate of $0.9 \%$. So compared to serial and parallel mode, proper combination of parallel and serial mode has given best accuracy.The Receiver Operating Characteristic (ROC) plot of FAR vs GAR of serial, parallel and hierarchical mode is represented in Figure 8.

Figure 9 shows the combined Receiver Operating Characteristic curve of unimodal and multimodal biometrics. From results it is observed that hierarchical mode of operation has given highest accuracy of $99 \%$ GAR at $0.01 \%$ FAR. Serial mode has given $93 \%$ GAR at $0.01 \%$ FAR and parallel mode has given accuracy of $92.5 \%$ at $0.01 \%$ FAR, which has given more accuracy than unimodal fingerprint and palmprint but less accuracy compare to unimodal iris. Unimodal iris system has given 96.5\% GAR at 0.01\%FAR. As all three traits are of different accuracy majority voting method has given less accuracy than iris. But with hierarchical mode there is improvement of $3 \%$ over most accurate iris unimodal system. So the results revealed that proper combination of traits in hierarchical mode can give the best performance as compared to serial and parallel mode even at decision level fusion.

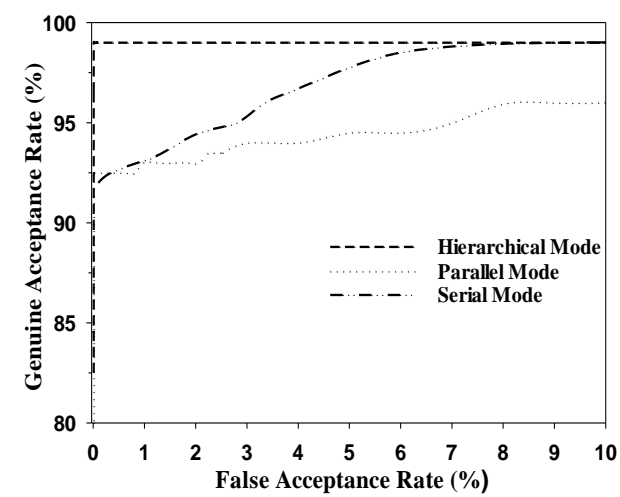

Figure 8. Performance of multimodal system at decision level fusion with parallel, serial and hierarchical mode of operation

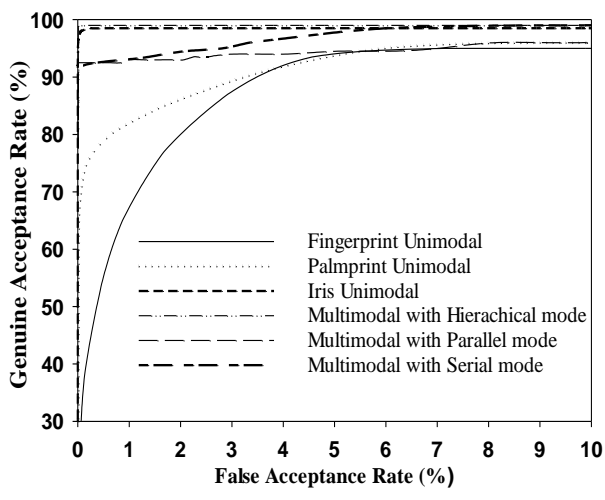

Figure 9. ROC curve of different biometric systems

\section{CONCLUSION}

The multimodal biometric verification system based on fingerprint, palmprint and iris at decision level fusion is proposed in this work. The system performance is measured at serial, parallel as well as hierarchical modes of operation. Experimental results indicate that the proper fusion of the different traits in parallel and serial mode in hierarchical manner using simple AND and OR rule gives more accuracy than only serial or parallel mode of operation. With hierarchical mode of operation system has achieved 99\% GAR at $0.01 \%$ FAR. Also with hierarchical mode there is an improvement of $\cong 3 \%$ in the accuracy as compare to most accurate unimodal i.e. iris unimodal system. So results suggest that if traits used in multimodal are having different accuracy then, their proper combination in hierarchical mode can give best accuracy than serial and parallel mode even at decision level fusion. 


\section{REFERENCES}

[1] Ross A, Jain A. Information Fusion in biometrics. Pattern Recognition Letters. 2003; (24): 2115-2125.

[2] R. Parimala and C. Jayakumar. TELKOMNIKA Indonesian Journal of Electrical Engineering. 2015; 15(3):591-596.

[3] Zhou J, Su G, Jiang C, Deng Y, Li C. A face and fingerprint identity authentication system based on multi-route detection.Neurocomputing. 2007; 70(4): 922-931.

[4] Hong L, Jain A K, Pankanti S. Can multibiometrics improve performance? AutoID 99. USA. 1999; 1: $59-64$.

[5] Monwar M, Gavrilova M L. Multimodal Biometric System Using Rank-Level Fusion Approach. IEEE Transactions on Systems, Man, and Cybernetics - Part B: Cybernetics. 2009; 39(4): 867-878.

[6] Jain A, Nadakumar K, Ross A. Score normalization in multimodal biometric system. Pattern Recognition. 2005; (38): 2270-2285.

[7] Jain A K, Ross A, Prabhakar S. An Introduction to Biometric Recognition. IEEE Transactions on Circuits and Systems for Video Technology. 2004; 14(1): 4-20.

[8] Vassilios C, Adrian G, Bor s, Loannis P. Multimodal Decision-Level Fusion for Person Authentication. IEEE Transactions on Systems, Man, and Cybernetics - Part A: Systems and Humans. 1999; 29(6): 674-680.

[9] Garg S N, Vig R, Gupta S. Multimodal Biometric System Based On Decision Level Fusion. IEEE International conference on Signal Processing, Communication, Power and Embedded System. 2016. pp. 753-758.

[10] Vijayalakshmi G V, Mohana C. A Multimodal Biometric Recognition System Based On Decision Level Fusion for User Authentication. ARPN Journal of Engineering and Applied Sciences. 2016; 11(23): 13856-13865.

[11] Paul P P, Gavrilova M L, Alhajj R. Decision Fusion for Multimodal Biometrics Using Social Network Analysis. IEEE Transactions on Systems, Man, and Cybernetics: Systems. 2014; 1-12.

[12] Benaliouche H, Touahria M. Comparative study of multimodal biometric recognition by fusion of iris and fingerprint. The Scientific World Journal. 2014; 2014:1-13.

[13] Ibrahim A. Saleh, Laheeb M. Alzoubiady. Decision Level Fusion of Iris and Signature Biometrics for Personal Identification using Ant Colony Optimization. International Journal of Engineering and Innovative Technology (IJEIT). 2014; 3(11): 35-42.

[14] Abdolahi M, Mohamadi M, Jafari M. Multimodal Biometric system Fusion Using Fingerprint and Iris with Fuzzy Logic. International Journal of Soft Computing and Engineering. 2013; 2(6): 504-510.

[15] Kumar A, Hanmandlu M, Sanghvi H, Gupta H M. Decision Level Biometric Fusion Using Ant Colony Optimization. IEEE 17th International Conference on Image Processing. 2010; 1: 3105-3108.

[16] Hanmandlu M, Kumar A, Madasu V K, Yarlagadda P. Fusion of Hand Based Biometrics using Particle Swarm optimization. IEEE Fifth International Conference on Information Technology: New Generations. 2008; 783-788.

[17] Akhtar Z, Fumera G, Marcialis G L, Roli F. Evaluation of serial and parallel multibiometric systems under spoofing attacks. IEEE. 2012; 283-288.

[18] Dinca L M, Hancke G P. Fall of One, the Rise of Many: A Survey on Multi-Biometric Fusion Methods. IEEE Access. 2017; 5(1): 6247-6289.

[19] Sanjekar P S, Saraf P D, Patil B D. Review on Core Point Detection Techniques in Fingerprint. International Journal Computer Applications. 2014; 1(3): 18-20.

[20] C. Hemalatha and E. Logashanmugam. Analysis of Different M-band Wavelet Filters for Face Recognition using Nearest Neighbour Classifier. Indonesian Journal of Electrical Engineering and Computer Science. 2018; 12(2):824-831.

[21] Bhatnagar G, Wu Q, Raman B. A New Fractional Random Wavelet Transform for Fingerprint Security. IEEE Trans. On Systems, Man, and Cybernetics-Part A: Systems and Humans. 2012; 42(1): 262-275.

[22] Haddate Ullah, Shahin Mahmud, Rubana Hoque Chowhury. Identification of Brain Disorders by Sub-band Decomposition of EEG signals and Measurement of Signal to Noice Ratio.Indonesian Journal of Electrical Engineering and Computer Science. 2016; 4(3):568-579.

[23] Sanjekar P S, Patil J B. Method of ROI Extraction for Palmprint. Indian Patent Application No. 201621044219 A, Filed on Dec. 2016; Published on Feb. 2017.

[24] Iris database: http://web.iitd.ac.in/ biometrics/Database_Iris.html.

[25] Fingerprint database: http://atvs.ii.uam.es/atvs/fvc2006.html.

[26] Palmprint database: http://biometrics.idealtest.org.

[27] Faddly Mazlan, Afdallyna Harun and Saifullzwan Suliman. Facial Recognition in Multimodal Biometrics system for Finger Disabled Applicants. Indonesian Journal of Electrical Engineering and Computer Science. 2017; 6(3): 638-645.

[28] Zanuy M F. Data Fusion in Biometrics. IEEE A\&E Systems Magazine. 2005; 34-38. 